Mathematical Models and Methods in Applied Sciences

Vol. 12, No. 5 (2002) 721-736

(C) World Scientific Publishing Company

\title{
ON THE MEASURE-THEORETIC FOUNDATIONS OF THE SECOND LAW OF THERMODYNAMICS
}

\author{
ALFREDO MARZOCCHI* and ALESSANDRO MUSESTI ${ }^{\dagger}$ \\ Dipartimento di Matematica, Università degli Studi di Brescia, \\ Via Valotti 9, I-25133 Brescia, Italy \\ *amarzocc@ing.unibs.it \\ †a.musesti@dmf.unicatt.it \\ Received 18 May 2001 \\ Revised 28 November 2001 \\ Communicated by N. Bellomo
}

\begin{abstract}
Balance laws of the type of entropy are treated in the framework of geometric measure theory, and a weak version, although conceptually simple, of the Second Law of Thermodynamics is introduced, allowing extensions to measure-valued entropy productions and to sets of finite perimeter as subbodies.
\end{abstract}

Keywords: Cauchy interactions; entropy; sets with finite perimeter.

AMS Subject Classification: 74A15, 80A05, 80A17

\section{Introduction}

In this paper we reconsider the standard formulation of the Second Law of Thermodynamics in the general framework of fields with divergence measure, using Geometric Measure Theory and some recent results on Cauchy interactions. To our knowledge, the first attempt to put the Second Law of Thermodynamics within the framework of Geometric Measure Theory is Gurtin, Williams, Ziemer. ${ }^{4}$ Recently, ${ }^{5}$ the present authors studied the balance of heat and generalized it to fields having divergence measure and measure-valued sources. Here, with respect to Ref. 5, more general balances are considered, involving inequalities and superadditive entropy production functions, as pointed out in Ref. 4. Two main improvements have been achieved: first, we obtain the existence of temperatures and the Clausius-Duhem inequality with flux vector fields having divergence measure, a degree of generality which seems to be the highest compatible with the representation results of general fluxes (see, for example, the discussion in Degiovanni, Marzocchi, Musesti ${ }^{1}$ ). To this end, we dropped the absolute continuity of the fluxes with respect to Lebesgue measure: this is not an idle generalization, since it is conceivable to have some form of concentrated heat or entropy fluxes. Second, and perhaps more important, we find that the usual statement of the Second Law of Thermodynamics has to be weakened 
in order to have the Clausius-Duhem inequality valid for almost all subbodies: in fact, a particularly simple class of multi-intervals is sufficient to extend the result on almost all subbodies of finite perimeter, i.e. on a very wide class. In the present context it appears that the class of multi-intervals is preferable to that of simple $n$-intervals, since the lack of additivity of involved quantities (namely, the entropy production) does not allow natural extensions from $n$-intervals.

After definiting our basic definitions in Sec. 2, we state the entropy inequality in Sec. 3 and obtain the existence of temperatures in Sec. 4. Section 5 is devoted to the extension result, and Sec. 6 contains some technical proofs.

\section{Basic Definitions}

In this section we recall the definitions and tools of Geometric Measure Theory which extend the usual notions of e.g. normal, boundary, smooth vector field, together with the main definitions we use in the sequel. For a deeper and more systematic introduction, we refer the reader to Federer ${ }^{2}$ or Ziemer. ${ }^{8}$

We denote by $\mathcal{L}^{n}$ the Lebesgue outer measure on $\mathbb{R}^{n}$ and by $\mathcal{H}^{k}$ the $k$-dimensional Hausdorff outer measure.

Let $M \subseteq \mathbb{R}^{n}$. By cl $M$ and int $M$ we denote the closure and the interior of $M$ in $\mathbb{R}^{n}$, respectively. When $M$ is a Borel set, we also denote by $\mathfrak{B}(M)$ the $\sigma$-algebra of Borel subsets of $M$.

Denoting by $\mathrm{B}_{r}(x)$ the open ball with center $x$ and radius $r$, we introduce

$$
M_{*}=\left\{x \in \mathbb{R}^{n}: \lim _{r \rightarrow 0^{+}} \frac{\mathcal{L}^{n}\left(\mathrm{~B}_{r}(x) \backslash M\right)}{\mathcal{L}^{n}\left(\mathrm{~B}_{r}(x)\right)}=0\right\}
$$

and

$$
\partial_{*} M=\mathbb{R}^{n} \backslash\left[M_{*} \cup\left(\mathbb{R}^{n} \backslash M\right)_{*}\right],
$$

(the so-called measure-theoretic interior and measure-theoretic boundary of $M$, respectively), which are Borel subsets of $\mathbb{R}^{n}$. If $M_{*}=M$, we say that $M$ is normalized.

Now let $x \in \partial_{*} M$. We call the unit exterior normal vector to $M$ at $x$ the vector $u_{x} \in \mathbb{R}^{n}$ such that $\left|u_{x}\right|=1$, and

$$
\begin{gathered}
\lim _{r \rightarrow 0^{+}} \frac{\mathcal{L}^{n}\left(\left\{\xi \in \mathrm{B}_{r}(x) \cap M:(\xi-x) \cdot u_{x}>0\right\}\right)}{\mathcal{L}^{n}\left(\mathrm{~B}_{r}(x)\right)}=0, \\
\lim _{r \rightarrow 0^{+}} \frac{\mathcal{L}^{n}\left(\left\{\xi \in \mathrm{B}_{r}(x) \backslash M:(\xi-x) \cdot u_{x}<0\right\}\right)}{\mathcal{L}^{n}\left(\mathrm{~B}_{r}(x)\right)}=0
\end{gathered}
$$

(no more than one such vector can exist). Hence we can define a bounded Borel map $\mathbf{n}^{M}: \partial_{*} M \rightarrow \mathbb{R}^{n}$, called the unit exterior normal to $M$, setting $\mathbf{n}^{M}(x)=u_{x}$ where it exists and $\mathbf{n}^{M}(x)=0$ otherwise.

We say that $M$ has finite perimeter if $\mathcal{H}^{n-1}\left(\partial_{*} M\right)<+\infty$ (this implies the $\mathcal{L}^{n}$-measurability of $\left.M\right)$. In that case, $\mathbf{n}^{M}(x) \neq 0$ for $\mathcal{H}^{n-1}$-a.e. $x \in \partial_{*} M$ and the 
Gauss-Green Theorem

$$
\int_{M} \mathbf{v} \cdot \operatorname{grad} f d \mathcal{L}^{n}=\int_{\partial_{*} M} f \mathbf{v} \cdot \mathbf{n}^{M} d \mathcal{H}^{n-1}-\int_{M} f \operatorname{div} \mathbf{v} d \mathcal{L}^{n}
$$

holds whenever $f: \mathbb{R}^{n} \rightarrow \mathbb{R}$ and $\mathbf{v}: \mathbb{R}^{n} \rightarrow \mathbb{R}^{n}$ are Lipschitz continuous with compact support (see Federer, ${ }^{2}$ Theorem 4.5.6 or Ziemer, ${ }^{8}$ Theorem 5.8.2).

Now let $\Omega$ be an open subset of $\mathbb{R}^{n}$. We denote by $\mathcal{L}_{\text {loc }}^{1}\left(\Omega ; \mathbb{R}^{n}\right)$ the set of Borel maps $\mathbf{v}: \Omega \rightarrow \mathbb{R}^{n}$ with $\int_{K}|\mathbf{v}| d \mathcal{L}^{n}<+\infty$ for any compact subset $K$ of $\Omega$, by $\mathcal{L}_{\text {loc },+}^{1}(\Omega)$ the set of Borel functions $h: \Omega \rightarrow[0,+\infty]$ with $\int_{K} h d \mathcal{L}^{n}<+\infty$ for every compact subset $K \subseteq \Omega$ and by $\mathfrak{M}(\Omega)$ the set of Borel measures $\mu: \mathfrak{B}(\Omega) \rightarrow[0,+\infty]$ finite on compact subsets of $\Omega$. Moreover, for $\mu, \eta \in \mathfrak{M}(\Omega)$ we will say that $\mu$ is absolutely continuous with respect to $\eta$, and write $\mu \ll \eta$, if

$$
\forall E \in \mathfrak{B}(\Omega): \eta(E)=0 \Longrightarrow \mu(E)=0 .
$$

Let $\mathbf{q} \in \mathcal{L}_{\text {loc }}^{1}\left(\Omega ; \mathbb{R}^{n}\right)$; we say that $\mathbf{q}$ has divergence measure, if div $\mathbf{q}$ is a distribution on $\Omega$ of order 0 , i.e. for every compact subset $K$ of $\Omega$ there exists a constant $c_{K}$ such that

$$
\left|\int_{\Omega} \mathbf{q} \cdot \operatorname{grad} f d \mathcal{L}^{n}\right| \leq c_{K} \max _{K}|f|
$$

for every $f \in C_{0}^{\infty}(\Omega)$ with supt $f \subseteq K$.

Throughout the remainder of this work, $B$ will denote a bounded normalized subset of $\mathbb{R}^{n}$ of finite perimeter, which we call, in the spirit of Šilhavý, ${ }^{6}$ a continuous body.

Definition 2.1. Let $\mathcal{M}$ be the collection of all normalized subsets of $B$ of finite perimeter. We set

$$
\begin{aligned}
& \mathcal{M}^{\mathrm{loc}}=\{A \in \mathcal{M}: \operatorname{cl} A \subseteq \operatorname{int} B\}, \\
& \mathcal{N}^{\text {loc }}=\mathcal{M}^{\text {loc }} \cup\left\{A \cup\left(\mathbb{R}^{n} \backslash B\right)_{*}: A \in \mathcal{M}^{\text {loc }}\right\}, \\
& \mathfrak{D}^{\text {loc }}=\left\{(A, C) \in \mathcal{M}^{\text {loc }} \times \mathcal{N}^{\text {loc }}: A \cap C=\emptyset\right\} .
\end{aligned}
$$

Let furthermore $h \in \mathcal{L}_{\text {loc, }+}^{1}(\operatorname{int} B)$ and $\nu \in \mathfrak{M}(\operatorname{int} B)$. We set

$$
\begin{aligned}
& \mathcal{M}_{h \nu}^{\text {loc }}=\left\{A \in \mathcal{M}^{\text {loc }}: \int_{\partial_{*} A} h d \mathcal{H}^{n-1}<+\infty, \nu\left(\partial_{*} A\right)=0\right\}, \\
& \mathcal{N}_{h \nu}^{\text {loc }}=\left\{C \in \mathcal{N}^{\text {loc }}:(C \cap B) \in \mathcal{M}_{h \nu}^{\text {loc }}\right\}, \\
& \mathfrak{D}_{h \nu}^{\text {loc }}=\mathfrak{D}^{\text {loc }} \cap\left(\mathcal{M}_{h \nu}^{\text {loc }} \times \mathcal{N}_{h \nu}^{\text {loc }}\right) .
\end{aligned}
$$

Definition 2.2. We say that $\mathcal{D} \subseteq \mathcal{M}^{\text {loc }}$ contains almost all of $\mathcal{M}^{\text {loc }}$, if $\mathcal{M}_{h \nu}^{\text {loc }} \subseteq \mathcal{D}$ for some $h \in \mathcal{L}_{\text {loc },+}^{1}(\operatorname{int} B)$ and $\nu \in \mathfrak{M}(\operatorname{int} B)$, and that a property $\pi$ holds almost everywhere in $\mathcal{M}^{\text {loc }}$, if the set

$$
\left\{A \in \mathcal{M}^{\text {loc }}: \pi(A) \text { is defined and } \pi(A) \text { holds }\right\}
$$


contains almost all of $\mathcal{M}^{\text {loc }}$.

We say that a subset $\mathcal{D}$ of $\mathfrak{D}^{\text {loc }}$ contains almost all of $\mathfrak{D}^{\text {loc }}$, if $\mathfrak{D}_{h \nu}^{\text {loc }} \subseteq \mathcal{D}$ for some $h \in \mathcal{L}_{\text {loc, }+}^{1}(\operatorname{int} B)$ and $\nu \in \mathfrak{M}(\operatorname{int} B)$, and that a property $\pi$ holds almost everywhere in $\mathfrak{D}^{\text {loc }}$, if the set

$$
\left\{(A, C) \in \mathfrak{D}^{\text {loc }}: \pi(A, C) \text { is defined and } \pi(A, C) \text { holds }\right\}
$$

contains almost all of $\mathfrak{D}^{\text {loc }}$.

For a full discussion on the concept of "almost all" etc. the reader is referred to Silhavý ${ }^{6}$ and Degiovanni, Marzocchi, Musesti. ${ }^{1}$ The rough idea is to leave out subbodies which may encounter singular sets of the measures involved, but not too many.

Definition 2.3. A grid $G$ is an ordered triple

$$
G=\left(x_{0},\left(e_{1}, \ldots, e_{n}\right), \hat{G}\right),
$$

where $x_{0} \in \mathbb{R}^{n},\left(e_{1}, \ldots, e_{n}\right)$ is an ordered orthonormal basis in $\mathbb{R}^{n}$ and $\hat{G}$ is a Borel subset of $\mathbb{R}$. If $G_{1}, G_{2}$ are two grids, we write $G_{1} \subseteq G_{2}$ if the first two components coincide and $\hat{G}_{1} \subseteq \hat{G}_{2}$. A grid $G$ is said to be full, if $\mathcal{L}^{1}(\mathbb{R} \backslash \hat{G})=0$.

Let $G$ be a grid; a subset $I$ of $\mathbb{R}^{n}$ is said to be an open $n$-dimensional $G$-interval, if

$$
I=\left\{x \in \mathbb{R}^{n}: a^{(j)}<\left(x-x_{0}\right) \cdot e_{j}<b^{(j)} \quad \forall j=1, \ldots, n\right\}
$$

for some $a^{(1)}, b^{(1)}, \ldots, a^{(n)}, b^{(n)} \in \hat{G}$. We set

$$
\mathcal{I}_{G}=\{I: I \text { is an open } n \text {-dimensional } G \text {-interval with } \operatorname{cl} I \subseteq \operatorname{int} B\} .
$$

The following proposition is a particular case of Proposition 4.5 in Degiovanni, Marzocchi, Musesti. ${ }^{1}$

Proposition 2.1. Let $x_{0} \in \mathbb{R}^{n}$ and $\left(e_{1}, \ldots, e_{n}\right)$ be an ordered orthonormal basis $\left(e_{1}, \ldots, e_{n}\right)$ in $\mathbb{R}^{n}$. Then for every $h \in \mathcal{L}_{\text {loc },+}^{1}(\operatorname{int} B)$ and $\nu \in \mathfrak{M}(\operatorname{int} B)$ there exists a full grid $G$ of the form $G=\left(x_{0},\left(e_{1}, \ldots, e_{n}\right), \hat{G}\right)$ such that $\mathcal{I}_{G} \subseteq \mathcal{M}_{h \nu}^{\text {loc }}$.

Definition 2.4. Given a grid $G$, a subset $P$ of $\mathbb{R}^{n}$ is said to be a $G$-figure, if $P=\left(\bigcup_{I \in \mathcal{F}} I\right)_{*}$, where $\mathcal{F}$ is a finite family of open $n$-dimensional $G$-intervals.

For any grid $G$, we set

$$
\begin{aligned}
\mathcal{P}_{G}= & \{P: P \text { is a } G \text {-figure with cl } P \subseteq \operatorname{int} B\}, \\
\mathfrak{P}_{G}= & \left\{(A, C) \in \mathfrak{D}: A, C \in \mathcal{P}_{G}, A \cap C=\emptyset\right\} \\
& \cup\left\{\left(A, C \cup\left(\mathbb{R}^{n} \backslash B\right)_{*}\right): A, C \in \mathcal{P}_{G}, A \cap C=\emptyset\right\} .
\end{aligned}
$$

The concepts of grid, $G$-figure etc. will be used in the "discrete" foundation of the Second Law later on. Now we give the definitions concerning interactions and superadditive functions defined on subbodies. 
Definition 2.5. Let $\mathcal{A} \subseteq \mathcal{N}^{\text {loc }}$. A function $F: \mathcal{A} \longrightarrow \mathbb{R}$ is superadditive if

$$
F\left(\left(A_{1} \cup A_{2}\right)_{*}\right) \geq F\left(A_{1}\right)+F\left(A_{2}\right)
$$

whenever $A_{1}, A_{2},\left(A_{1} \cup A_{2}\right)_{*} \in \mathcal{A}$ and $A_{1} \cap A_{2}=\emptyset$. If the above relation holds with the equal sign, $F$ is said to be additive.

Let $\mathcal{D} \subseteq \mathfrak{D}^{\text {loc }}$. We say that a function $F: \mathcal{D} \longrightarrow \mathbb{R}$ is biadditive if the functions

$$
\begin{aligned}
& F(\cdot, C):\left\{A^{\prime} \in \mathcal{M}:\left(A^{\prime}, C\right) \in \mathcal{D}\right\} \longrightarrow \mathbb{R}, \\
& F(A, \cdot):\left\{C^{\prime} \in \mathcal{N}:\left(A, C^{\prime}\right) \in \mathcal{D}\right\} \longrightarrow \mathbb{R},
\end{aligned}
$$

are additive for every $(A, C) \in \mathcal{D}$.

Definition 2.6. Let $\mathcal{D} \subseteq \mathfrak{D}^{\text {loc }}$ be a set containing almost all of $\mathfrak{D}^{\text {loc }}$ and consider a function $I: \mathcal{D} \longrightarrow \mathbb{R}$. We say that $I$ is a Cauchy interaction, if the following properties hold:

(a) $I$ is biadditive;

(b) there exist $h \in \mathcal{L}_{\text {loc },+}^{1}($ int $B), \eta \in \mathfrak{M}(\operatorname{int} B \times \operatorname{int} B)$ and $\eta_{e} \in \mathfrak{M}(\operatorname{int} B)$ such that the inequality

$$
|I(A, C)| \leq \begin{cases}\int_{\partial_{*} A \cap \partial_{*} C} h d \mathcal{H}^{n-1}+\eta(A \times C) & \text { if } C \subseteq B \\ \int_{\partial_{*} A \cap \partial_{*} C} h d \mathcal{H}^{n-1}+\eta(A \times(C \cap B))+\eta_{e}(A) & \text { otherwise }\end{cases}
$$

holds almost everywhere in $\mathfrak{D}^{\text {loc }}$.

The last definition, motivated by a choice already made in Gurtin, Williams, Ziemer, ${ }^{4}$ covers the possibility that the exterior of the body, considered as a whole, has a bulk interaction with its parts. If it is not the case, it is enough to set $\eta_{e}=0$.

Definition 2.7. A Cauchy interaction $I$ is said to be balanced, if there exists a measure $\lambda \in \mathfrak{M}($ int $B)$ such that

$$
\partial_{*} A \subseteq \partial_{*} C \Longrightarrow|I(A, C)| \leq \lambda(A)
$$

on almost all of $\mathfrak{D}^{\text {loc }}$.

This concept is a generalization of the idea for a quantity to obey a balance law (see Refs. 4 and 5).

If we take $\mathcal{D}=\mathfrak{P}_{G}$ for some grid $G$ and require $I$ to satisfy Definitions 2.6 and 2.7 only on $\mathfrak{P}_{G}$ for suitable $h, \eta, \lambda$, we shall call the function $I$ a $G$-interaction. This means that we have an interaction defined only on special subbodies, the $G$-figures. In Marzocchi, Musesti ${ }^{5}$ (Corollary 8.1) it is proved that if $G$ is a full grid, then a $G$-interaction can be extended in an essentially unique way to a balanced Cauchy interaction. 
Remark 2.1. When $I$ is balanced, it is possible to enlarge the domain of $I$ to the set

$$
\mathfrak{D}_{h \nu}^{\text {loc }} \cup\left\{(A, C): A \in \mathcal{M}_{h \nu}^{\text {loc }}, C \text { is normalized, }\left(\mathbb{R}^{n} \backslash C\right)_{*} \in \mathcal{M}_{h \nu}^{\text {loc }}, A \cap C=\emptyset\right\}
$$

for suitable $h$ and $\nu$ (cf. Theorem 7.4 in Ref. 5). In particular the interaction between a subbody $A$ and its exterior, $I\left(A,\left(\mathbb{R}^{n} \backslash A\right)_{*}\right)$, is defined for almost every $A \in \mathcal{M}^{\text {loc }}$.

\section{Balance of Entropy}

During a thermodynamic process, amounts of entropy (as well as of other quantities, such as heat) transfer from a subbody to another, so that we can model this transfer by means of a Cauchy interaction. In view of a balance law, we call entropy transfer a balanced Cauchy interaction $M(A, C)$ which can be interpreted as the amount of entropy that the subbody $C$ transfers to $A$. It is therefore a biadditive function. However, due to the peculiar nature of entropy, an extra amount can be produced in the process. Hence, we introduce an entropy production term, i.e. a real-valued function $\Gamma$ defined on almost all of $\mathcal{M}^{\text {loc }}$.

In the standard frame, a balance law of the form

$$
\dot{S}(A)=M\left(A, A^{e}\right)+\Gamma(A)
$$

is introduced, where $A^{e}$ denotes the exterior of the subbody $A$ and $\dot{S}$ is the rate of change of internal entropy, and a local form for regular fields is deduced. For instance, if all expressions involved make sense, one can write

$$
\dot{S}(A)=\int_{A} \dot{s} d \mathcal{L}^{n}, \quad M\left(A, A^{e}\right)=\int_{\partial_{*} A} \mathbf{j} \cdot \mathbf{n}^{A} d \mathcal{H}^{n-1}, \quad \Gamma(A)=\int_{A} g d \mathcal{L}^{n}
$$

and get the usual differential equality

$$
\dot{s}=\operatorname{div} \mathbf{j}+g .
$$

The usual interpretation of the principle of increase of entropy implies $\Gamma \geq 0$; Moreover, using the biadditivity of $M$, it is not hard to see that

$$
\dot{S}(A \cup C)-\dot{S}(A)-\dot{S}(C)=-(M(A, C)+M(C, A))+\Gamma(A \cup C)-\Gamma(A)-\Gamma(C) .
$$

If the terms $M(A, C)$ and $M(C, A)$ are surface integrals, then their sum vanishes and

$$
\dot{S}(A \cup C)-\dot{S}(A)-\dot{S}(C)=\Gamma(A \cup C)-(\Gamma(A)+\Gamma(C)) .
$$

In Truesdell ${ }^{7}$ (Appendix G4), Gurtin and Williams called the left side binding entropy; since in some cases it may be strictly positive (see again Truesdell ${ }^{7}$ and Sec. 6 of Ref. 4), we will suppose in the sequel that $\Gamma$ is superadditive. Moreover, we do not want $\Gamma$ to be necessarily absolutely continuous with respect to the volume measure.

Definition 3.1. An entropy production is a real-valued function $\Gamma$ defined on almost all of $\mathcal{M}^{\text {loc }}$ such that 
(a) $\Gamma \geq 0$ on almost all of $\mathcal{M}^{\text {loc; }}$

(b) there exists $\eta \in \mathfrak{M}(\operatorname{int} B)$ with

$$
\Gamma(A) \leq \eta(A)
$$

for almost every $A \in \mathcal{M}^{\text {loc; }}$

(c) $\Gamma$ is superadditive.

Of course, this definition belongs to every situation in which a balance law holds with an extra production term. Since $\Gamma$ is positive, from (c) it follows that it is monotone, i.e.

$$
A_{1} \subseteq A_{2} \Rightarrow \Gamma\left(A_{1}\right) \leq \Gamma\left(A_{2}\right) .
$$

The following theorem states the main property of entropy productions.

Theorem 3.1. If $\Gamma$ is an entropy production, then there exists a Borel function $g:$ int $B \rightarrow[0,+\infty)$ such that:

(i) for almost every $A \in \mathcal{M}^{\text {loc }}$,

$$
\int_{A} g d \eta \leq \Gamma(A)
$$

(ii) $g$ is maximal, in the sense that if $G$ is a full grid such that $\Gamma$ is defined on $\mathcal{P}_{G}$ and $f:$ int $B \rightarrow[0,+\infty)$ is a Borel function satisfying

$$
\forall P \in \mathcal{P}_{G}: \int_{P} f d \eta \leq \Gamma(P),
$$

then $f(x) \leq g(x)$ for $\eta$-almost all $x \in \operatorname{int} B$.

Moreover, such a $g$ is uniquely determined $\eta$-a.e.

This is a plain consequence of Theorem 6.2 , which we postpone to the last section. It is quite a delicate theorem in Measure Theory; therefore, it is somewhat technical.

In particular, the maximal density $g$ produces a maximal measure

$$
\gamma(A)=\int_{A} g d \eta
$$

with $\gamma(A) \leq \Gamma(A)$ on almost all of $\mathcal{M}^{\text {loc }}$.

Definition 3.2. The maximal measure $\gamma$ is called the optimal entropy production.

Now we recall the integral representation of the entropy transfer $M$ : by Theorem 7.4 of Ref. 5 we have

$$
M(A, C)= \begin{cases}\int_{A \times C} k d \alpha+\int_{\partial_{*} A \cap \partial_{*} C} \mathbf{j} \cdot \mathbf{n}^{A} d \mathcal{H}^{n-1} & \text { if } C \subseteq B, \\ \int_{A \times(C \cap \operatorname{int} B)} k d \alpha+\int_{A} k_{e} d \alpha_{e}+c \int_{\partial_{*} A \cap \partial_{*} C} \mathbf{j} \cdot \mathbf{n}^{A} d \mathcal{H}^{n-1} & \text { otherwise, }\end{cases}
$$


on almost all of $\mathfrak{D}^{\text {loc }}$, where $\alpha \in \mathfrak{M}(\operatorname{int} B \times \operatorname{int} B), \alpha_{e} \in \mathfrak{M}(\operatorname{int} B)$, the Borel functions $k: \operatorname{int} B \times \operatorname{int} B \rightarrow \mathbb{R}$ and $k_{e}: \operatorname{int} B \rightarrow \mathbb{R}$ are such that $|k|=1 \alpha$-a.e. and $\left|k_{e}\right|=1 \alpha_{e}$-a.e. respectively, $\mathbf{j} \in \mathcal{L}_{\text {loc }}^{1}\left(\operatorname{int} B ; \mathbb{R}^{n}\right)$ has divergence measure.

In (3.1) $k$ represents the density of the bulk entropy transfer, while $\mathbf{j}$ is the surface density of the entropy flux. Finally, $k_{e}$ takes into account possible bulk entropy exchanges with the exterior of the body.

For almost every $A \in \mathcal{M}^{\text {loc }}$ we define

$$
\begin{aligned}
M(A, A) & :=\int_{A \times A} k d \alpha \\
M\left(A, \mathbb{R}^{n}\right) & :=M\left(A,\left(\mathbb{R}^{n} \backslash A\right)_{*}\right)+M(A, A) \\
& =\int_{A \times \operatorname{int} B} k d \alpha+\int_{A} k_{e} d \alpha_{e}+\int_{\partial_{*} A} \mathbf{j} \cdot \mathbf{n}_{\partial_{*} A} d \mathcal{H}^{n-1} .
\end{aligned}
$$

Notice that, in order to speak of $\left(\mathbb{R}^{n} \backslash A\right)_{*}$ as a subbody, we rely on Remark 2.1.

Now, combine the entropy production and the entropy transfer: clearly the measure $\gamma+M\left(\cdot, \mathbb{R}^{n}\right)$ is additive and bounded by a Radon measure on almost all of $\mathcal{M}^{\text {loc }}$, so it can be represented by a measure $\sigma \in \mathfrak{M}(\operatorname{int} B)$ and a Borel function $u$ with $|u(x)|=1$ for $\sigma$-a.e. $x \in \operatorname{int} B$, i.e.

$$
\gamma(A)+M\left(A, \mathbb{R}^{n}\right)=\int_{A} u d \sigma
$$

on almost all of $\mathcal{M}^{\text {loc }}$. Since $\gamma$ is positive, it is obvious that $\int_{A} u d \sigma \geq M\left(A, \mathbb{R}^{n}\right)$. Hence,

$$
\int_{A} u d \sigma \geq \int_{A} \operatorname{div} \mathbf{j}+\int_{A \times \operatorname{int} B} k d \alpha+\int_{A} k_{e} d \alpha_{e}
$$

on almost all of $\mathcal{M}^{\text {loc }}$. This is the measure-theoretic version of the entropy inequality.

Remark 3.1. Clearly we have

$$
\Gamma(A)+M\left(A,\left(\mathbb{R}^{n} \backslash A\right)_{*}\right) \geq \gamma(A)+M\left(A,\left(\mathbb{R}^{n} \backslash A\right)_{*}\right) .
$$

The quantity $\Gamma(A)+M\left(A,\left(\mathbb{R}^{n} \backslash A\right)_{*}\right)$ is usually interpreted as the rate of change of the internal entropy of the subbody $A$. We can hence look at $\gamma(A)+M\left(A,\left(\mathbb{R}^{n} \backslash A\right)_{*}\right)$ as the optimal rate of change of internal entropy of $A$, accordingly to Definition 3.2.

\section{Existence of Temperatures}

In this section we suppose that, beyond the entropy transfer, there exists a balanced Cauchy interaction $H$ which we refer to as the heat transfer. By Theorem 7.4 of Ref. 5 , there exist $b, b_{e}, \mu, \mu_{e}, \mathbf{q}$ (with rôles analogous to the corresponding list for entropy) such that 
$H(A, C)= \begin{cases}\int_{A \times C} b d \mu+\int_{\partial_{*} A \cap \partial_{*} C} \mathbf{q} \cdot \mathbf{n}^{A} d \mathcal{H}^{n-1} & \text { if } C \subseteq B, \\ \int_{A \times(C \cap \operatorname{int} B)} b d \mu+\int_{A} b_{e} d \mu_{e}+\int_{\partial_{*} A \cap \partial_{*} C} \mathbf{q} \cdot \mathbf{n}^{A} d \mathcal{H}^{n-1} & \text { otherwise },\end{cases}$ on almost all of $\mathfrak{D}^{\text {loc }}$.

Physical evidence shows that when there is no heat transfer, no entropy can be transferred. Therefore, the following assumption is a crucial part of the Second Law of Thermodynamics. Without it, one could not have a link between temperature and entropy (or even the concept of temperature).

Assumption 4.1. Let $h \in \mathcal{L}_{\text {loc },+}^{1}($ int $B), \nu \in \mathfrak{M}($ int $B)$ be such that the representation formulas for $M$ and $H$ hold on $\mathfrak{D}_{h \nu}^{\text {loc }}$. Given $(A, C) \in \mathfrak{D}_{h \nu}^{\text {loc }}$, if $H(\hat{A}, \hat{C})=0$ for every $(\hat{A}, \hat{C}) \in \mathfrak{D}_{h \nu}^{\text {loc }}$ such that $\hat{A} \subseteq A, \hat{C} \subseteq C$, then $M(A, C)=0$.

The goal of this section is to prove that the measures associated with $M$ in the representation (3.1) are absolutely continuous with respect to those associated with $H$; in this way, we will find three functions playing the rôle of temperature in a generalization of the classical Clausius-Duhem inequality. This is not trivial, since we need to associate in some way a measure of $A \times C$ to the surface integrals.

From now on, we will use the notation $E^{*}=E \cup \partial_{*} E$; this is the measuretheoretic closure of $E$. First of all, we state a slight variant of Theorem 7.4 of Ref. 5 for balanced Cauchy interactions in terms of the measure-theoretic closed sets $E^{*}$.

Theorem 4.1. Let $H$ be an arbitrary balanced Cauchy interaction and let $\mathbf{q}$, $b, b_{e}, \mu, \mu_{e}$ be as in Theorem 7.4 of Ref. 5. Then the formula

$H(A, C)= \begin{cases}\int_{A^{*} \times C^{*}} b d \mu+\int_{\partial_{*} A^{*} \cap \partial_{*} C^{*}} \mathbf{q} \cdot \mathbf{n}^{A} d \mathcal{H}^{n-1} & \text { if } C \subseteq B, \\ \int_{A^{*} \times(C \cap \operatorname{int} B)^{*}} b d \mu+\int_{A^{*}} b_{e} d \mu_{e}+\int_{\partial_{*} A^{*} \cap \partial_{*} C^{*}} \mathbf{q} \cdot \mathbf{n}^{A} d \mathcal{H}^{n-1} & \text { otherwise, }\end{cases}$ holds on almost all of $\mathfrak{D}^{\text {loc }}$.

Proof. Clearly, for every $A \in \mathfrak{B}$ (int $B$ ) we have that $A^{*}$ is the disjoint union of $A_{*}$ and $\partial_{*} A$. Since we can choose $h \in \mathcal{L}_{\text {loc },+}^{1}(\operatorname{int} B)$ and $\nu \in \mathfrak{M}(\operatorname{int} B)$ in a way such that $\mu\left(\partial_{*} A \times \operatorname{int} B\right)=\mu\left(\operatorname{int} B \times \partial_{*} C\right)=\mu_{e}\left(\partial_{*} A \cup \partial_{*} C\right)=0$ for every $(A, C) \in \mathfrak{D}_{h \nu}^{\text {loc }}$, it is apparent that the volume integrals involving $\partial_{*} A$ and $\partial_{*} C$ vanish. Finally, notice that $\partial_{*} A=\partial_{*} A^{*}$.

Now let $(A, C) \in \mathfrak{D}_{h \nu}^{\text {loc }}$ with $C \subseteq B$ and let $f: \operatorname{int} B \rightarrow \operatorname{int} B \times \operatorname{int} B$ be such that $f(x)=(x, x)$; we define two real-valued measures $m_{1}, m_{2}$ on $\mathfrak{B}(A \times C)$ setting

$$
m_{1}(E)=\sqrt{2} \int_{E} \mathbf{q}\left(f^{-1}(x, y)\right) \cdot \mathbf{n}^{A}\left(f^{-1}(x, y)\right) d \mathcal{H}^{n-1}(x, y),
$$




$$
m_{2}(E)=\sqrt{2} \int_{E} \mathbf{j}\left(f^{-1}(x, y)\right) \cdot \mathbf{n}^{A}\left(f^{-1}(x, y)\right) d \mathcal{H}^{n-1}(x, y) .
$$

The previous definitions allow to express the surface integrals associated to $H$ and $M$, denoted by $H_{c}$ and $M_{c}$ respectively, in terms of the measures $m_{i}$ : by a change of variables it results that for every $(\hat{A}, \hat{C}) \in \mathfrak{D}_{h \nu}^{\text {loc }}$ with $\hat{A} \subseteq A$ and $\hat{C} \subseteq C$, we have

$$
H_{c}(\hat{A}, \hat{C})=m_{1}\left(\hat{A}^{*} \times \hat{C}^{*}\right), \quad M_{c}(\hat{A}, \hat{C})=m_{2}\left(\hat{A}^{*} \times \hat{C}^{*}\right) .
$$

Details are omitted for the sake of brevity.

Let $p_{1}, p_{2}$ be the densities of $m_{1}, m_{2}$, respectively. Combining this fact with Theorem 4.1, it follows that

$$
\begin{aligned}
& H(\hat{A}, \hat{C})=\int_{\hat{A}^{*} \times \hat{C}^{*}} b d \mu+\int_{\hat{A}^{*} \times \hat{C}^{*}} p_{1} d\left|m_{1}\right|, \\
& M(\hat{A}, \hat{C})=\int_{\hat{A}^{*} \times \hat{C}^{*}} k d \alpha+\int_{\hat{A}^{*} \times \hat{C}^{*}} p_{2} d\left|m_{2}\right|,
\end{aligned}
$$

for every $(\hat{A}, \hat{C}) \in \mathfrak{D}_{h \nu}^{\text {loc }}$ with $\hat{A} \subseteq A, \hat{C} \subseteq C$. Moreover, the supports of $\left|m_{1}\right|$ and $\left|m_{2}\right|$ lie in $\left(\partial_{*} A \cap \partial_{*} C\right) \times\left(\partial_{*} A \cap \partial_{*} C\right)$, while the supports of $\mu$ and $\alpha$ lie in $A \times C \backslash\left(\left(\partial_{*} A \cap \partial_{*} C\right) \times\left(\partial_{*} A \cap \partial_{*} C\right)\right)$.

By Assumption 4.1, we have that $\alpha+\left|m_{2}\right| \ll \mu+\left|m_{1}\right|$; since the supports are disjoint, it follows that

$$
\alpha \ll \mu, \quad\left|m_{2}\right| \ll\left|m_{1}\right|
$$

and there exist two Borel functions $\theta_{A, C}, d_{A, C}: A \times C \rightarrow \mathbb{R}$ such that

$$
M(\hat{A}, \hat{C})=\int_{\hat{A}^{*} \times \hat{C}^{*}} \theta_{A, C} b d \mu+\int_{\hat{A}^{*} \times \hat{C}^{*}} d_{A, C} p_{1} d\left|m_{1}\right|
$$

for every $(\hat{A}, \hat{C}) \in \mathfrak{D}_{h \nu}^{\text {loc }}$ with $\hat{A} \subseteq A, \hat{C} \subseteq C$. Our choice of having fixed $A$ and $C$ is not restrictive: it is readily seen that for $\left(A_{1}, C_{1}\right),\left(A_{2}, C_{2}\right) \in \mathfrak{D}_{h \nu}^{\text {loc }}$ one has

$$
\begin{array}{ll}
\theta_{A_{1} \cap A_{2}, C_{1} \cap C_{2}}=\left.\theta_{A_{1}, C_{1}}\right|_{\left(A_{1} \cap A_{2}\right) \times\left(C_{1} \cap C_{2}\right)}=\left.\theta_{A_{2}, C_{2}}\right|_{\left(A_{1} \cap A_{2}\right) \times\left(C_{1} \cap C_{2}\right)} \quad \mu \text {-a.e. } \\
d_{A_{1} \cap A_{2}, C_{1} \cap C_{2}}=\left.d_{A_{1}, C_{1}}\right|_{\left(A_{1} \cap A_{2}\right) \times\left(C_{1} \cap C_{2}\right)}=\left.d_{A_{2}, C_{2}}\right|_{\left(A_{1} \cap A_{2}\right) \times\left(C_{1} \cap C_{2}\right)} \quad\left|m_{1}\right| \text {-a.e. }
\end{array}
$$

Since $\theta_{A, C}$ and $d_{A, C}$ agree on intersections, they can be extended in a unique (almost everywhere) way to $\theta, d: \operatorname{int} B \times \operatorname{int} B \rightarrow \mathbb{R}$.

We are now in position to introduce the notion of the reciprocal of the contact temperature, setting $\theta_{c}=d \circ f$; we have that

$$
M_{c}(A, C)=\int_{\partial_{*} A \cap \partial_{*} C} \theta_{c} \mathbf{q} \cdot \mathbf{n}^{A} d \mathcal{H}^{n-1}
$$

on almost all of $\mathfrak{D}^{\text {loc }} .^{\text {a }}$ 
On the other hand, if $\left(A,\left(\mathbb{R}^{n} \backslash B\right)_{*}\right) \in \mathfrak{D}_{h \nu}^{\text {loc }}$ we can repeat in an easier way the same procedure, getting a Borel function $\theta_{e}: \operatorname{int} B \rightarrow \mathbb{R}$ such that

$$
M\left(A,\left(\mathbb{R}^{n} \backslash B\right)_{*}\right)=\int_{A^{*}} \theta_{e} b_{e} d \mu_{e} .
$$

Finally we have

$$
M(A, C)=\int_{\partial_{*} A \cap \partial_{*} C} \theta_{c} \mathbf{q} \cdot \mathbf{n}^{A} d \mathcal{H}^{n-1}+\int_{A^{*} \times C^{*}} \theta b d \mu+\int_{A^{*}} \theta_{e} b_{e} d \mu_{e}
$$

on almost all of $\mathfrak{D}^{\text {loc }}$. The meaning of the integrands in (4.1) is that of reciprocal of absolute temperatures related to interactions: $1 / \theta_{c}$ is the usual one, since it is associated to the contact part, while the others, associated to bulk and external bulk heat transfer, appear in some applications, such as the theory of radiative transfer. Notice that we introduce $\theta_{c}$ and not its reciprocal: indeed, when $\theta_{c}$ vanishes the usual temperature does not exist.

We can now restate the entropy inequality of the previous section, involving the densities of the heat interaction:

$$
\begin{aligned}
\int_{A} u d \sigma \geq & \int_{\partial_{*} A} \theta_{c}(x) \mathbf{q}(x) \cdot \mathbf{n}^{A}(x) d \mathcal{H}^{n-1}(x) \\
& +\int_{A \times \operatorname{int} B} \theta(x, y) b(x, y) d \mu(x, y)+\int_{A} \theta_{e}(x) b_{e}(x) d \mu_{e}(x)
\end{aligned}
$$

on almost all of $\mathcal{M}^{\text {loc }}$, which is a generalization of the integral form of the ClausiusDuhem inequality.

\section{A Weaker Form of the Second Law}

The aim of this section is to introduce the Second Law of Thermodynamics in a sort of "discrete" context and deduce all results proved up to here.

In Sec. 8 of Marzocchi, Musesti, ${ }^{5}$ a notion of balanced Cauchy interaction defined only on almost all $n$-intervals is introduced, and an extension theorem is proved. In that context, the choice between multi-intervals or $n$-intervals fell on the second class, since the biadditivity bears immediately an extension to the first. Here, on the contrary, the entropy production is only superadditive and there is no natural way to extend it. Because of this fact, the class of almost all multi-intervals seems to be the simplest natural class of subbodies which gives enough information to extend the notions to almost all normalized sets of finite perimeter, at least in the framework of Thermodynamics.

Let $G$ be a full grid, and consider two $G$-interactions $M, H: \mathfrak{P}_{G} \rightarrow \mathbb{R}$. These functions represent the entropy transfer and the heat transfer, respectively. As remarked in Sec. 2, in Marzocchi, Musesti ${ }^{5}$ it is proved that $M$ and $H$ can be extended in an essentially unique way to balanced Cauchy interactions.

Then consider a function $\Gamma: \mathcal{P}_{G} \rightarrow \mathbb{R}$, which takes the place of the entropy production of Sec. 3. We restate the Second Law of Thermodynamics in the following way. 
Axiom 5.1. (Second Law of Thermodynamics) For a full grid $G$ there exist two $G$-interactions $M, H$ and a function $\Gamma: \mathfrak{P}_{G} \rightarrow \mathbb{R}$ such that:

(i) $\Gamma \geq 0$;

(ii) there exists $\eta \in \mathfrak{M}(\operatorname{int} B)$ with

$$
\Gamma(P) \leq \eta(P)
$$

for every $P \in \mathcal{P}_{G}$;

(iii) $\Gamma$ is superadditive;

(iv) given $(A, C) \in \mathfrak{P}_{G}$, we have $M(A, C)=0$ whenever $H(\hat{A}, \hat{C})=0$ for every $(\hat{A}, \hat{C}) \in \mathfrak{P}_{G}$ such that $\hat{A} \subseteq A, \hat{C} \subseteq C$.

Clearly, this is a weaker version of the Second Law of Thermodynamics, since each requirement involves only $G$-figures. The following theorem shows that this axiom is sufficient to prove the validity of the Clausius-Duhem inequality in its general form. This is our main result.

Theorem 5.1. If Axiom 5.1 holds, then (4.2) holds on almost all of $\mathcal{M}^{\text {loc }}$.

Proof. Theorem 6.2 below allows to find a unique optimal entropy production $\gamma$ such that (3.2) holds on almost all of $\mathcal{M}^{\text {loc }}$.

Moreover, by means of (iv) of Axiom 5.1 we can again prove that $\alpha \ll \mu$ and $\left|m_{1}\right| \ll\left|m_{2}\right|$ in Sec. 4 .

Once more we stress the fact that testing the validity of the Second Law only on almost all multi-intervals, we obtain the same results as testing it on almost all of $\mathcal{M}^{\text {loc }}$ and $\mathfrak{D}^{\text {loc }}$.

\section{Superadditive Functions}

This section is essentially devoted to proving Theorem 3.1. In order to do so, we need some preliminary material.

Let $C(x, r)$ denote the closed cube with center $x$ and edge $2 r$. Given a full grid $G$, we denote by $\mathcal{C}_{G}$ the set of all closed cubes $C(x, r)$ such that $x \in \operatorname{int} B, r>0$ and $\operatorname{int} C(x, r) \in \mathcal{I}_{G}$.

Remark 6.1. (Geometric property) For any $r>0$ there exists a maximal number of closed cubes with edges greater than $r$ that do not contain the center of each other and such that all of them intersect a given cube having edge $r$.

Definition 6.1. A subfamily $\mathcal{C}$ of $\mathcal{C}_{G}$ is fine with respect to a set $E \subseteq \operatorname{int} B$, if every $x \in E$ is the center of an element $C \in \mathcal{C}$ and

$$
\inf \{r: \mathrm{C}(x, r) \in \mathcal{C}\}=0
$$

for every $x \in E$. 
The following, usually known as the Besicovitch Theorem, is a standard tool in Measure Theory and strongly relies on Remark 6.1. For a proof the reader is referred to Federer $^{2}$ 2.8.15.

Theorem 6.1. (Besicovitch Theorem) Let $\eta \in \mathfrak{M}(\operatorname{int} B), E \in \mathfrak{B}(\operatorname{int} B)$ with $\eta(E)<+\infty$ and $\mathcal{C}$ be a subfamily of $\mathcal{C}_{G}$ which is fine with respect to $E$.

Then for every open set $A \subseteq \operatorname{int} B$ with $E \subseteq A$, there exists a countable disjoint subfamily $\mathcal{G}$ of $\mathcal{C}$ such that

$$
\bigcup_{C \in \mathcal{G}} C \subseteq A, \quad \eta\left(E \backslash \bigcup_{C \in \mathcal{G}} C\right)=0 .
$$

The previous result also applies to the case of open normalized $n$-intervals and normalized unions, by choosing a suitable full grid $G$, as stated below.

Corollary 6.1. Let $\eta \in \mathfrak{M}(\operatorname{int} B)$ and $E \in \mathfrak{B}(\operatorname{int} B)$ with $\eta(E)<+\infty$. Let $G$ be a full grid such that $\mathcal{I}_{G} \subseteq \mathcal{M}_{0 \eta}^{\text {loc b }}$, and $\mathcal{C}$ be a subfamily of $\mathcal{C}_{G}$ which is fine with respect to $E$.

Then for every open normalized set $A \in \operatorname{int} B$ with $E \subseteq A$, there exists a countable disjoint subfamily $\left(I_{k}\right) \subseteq \mathcal{I}_{G}$ such that

$$
\left(\bigcup_{k \in \mathbb{N}} I_{k}\right)_{*} \subseteq A, \quad \eta\left(E \backslash\left(\bigcup_{k \in \mathbb{N}} I_{k}\right)_{*}\right)=0 .
$$

Proof. Let $\mathcal{G}=\left\{C_{k}: k \in \mathbb{N}\right\}$ be a countable subfamily of $\mathcal{C}$ as in Theorem 6.1 and set $I_{k}=\operatorname{int} C_{k}$. Then the family $\left(I_{k}\right)$ is contained in $\mathcal{I}_{G}$ and, keeping into account that $A$ is normalized, one has

$$
\left(\bigcup_{k \in \mathbb{N}} I_{k}\right)_{*} \subseteq A
$$

Moreover, $\eta \in \mathcal{M}_{0 \eta}^{\text {loc }}$ implies that

$$
\eta\left(E \backslash\left(\bigcup_{k \in \mathbb{N}} I_{k}\right)_{*}\right)=\eta\left(E \backslash \bigcup_{k \in \mathbb{N}} I_{k}\right)=0
$$

and the proof is complete.

Let now $\mathcal{A} \subseteq \mathcal{M}^{\text {loc }}$ and $\Gamma: \mathcal{A} \rightarrow[0,+\infty)$ be a superadditive function. We suppose that there exist a full grid $G$ and a measure $\eta \in \mathfrak{M}(\operatorname{int} B)$ such that $\mathcal{P}_{G} \subseteq \mathcal{A}, \mathcal{P}_{G} \subseteq \mathcal{M}_{0 \eta}^{\text {loc }}$ and $\Gamma \leq \eta$ on $\mathcal{P}_{G}$. Moreover, we suppose that $\mathcal{A}$ is closed by finite intersection. Note that if $\mathcal{A}=\mathcal{M}_{h \nu}^{\text {loc }}$ for suitable $h$ and $\nu$, as in Sec. 3 , or $\mathcal{A}=\mathcal{P}_{G}$ as in Sec. 5, then in any case it satisfies the previous requirement.

${ }^{\mathrm{b}}$ Clearly, $0 \eta$ means $h=0, \eta \in \mathfrak{M}(\operatorname{int} B)$. 
Now we set

$$
g(x)=\inf _{\rho>0} \sup \left(\{0\} \cup\left\{\frac{\Gamma(I)}{\eta(I)}: I \in \mathcal{C}_{G}, x \in I, \operatorname{diam} I<\rho, \eta(I)>0\right\}\right) .
$$

Lemma 6.1. The function $g:$ int $B \rightarrow[0,+\infty)$ is Borel and bounded.

Proof. As $\Gamma \leq \eta$, it is clear that $0 \leq g(x) \leq 1$, hence it is bounded. Let us define, for $\rho>0$,

$$
\delta_{\rho}(x)=\sup \left(\{0\} \cup\left\{\frac{\Gamma(I)}{\eta(I)}: I \in \mathcal{C}_{G}, x \in I, \operatorname{diam} I<\rho, \eta(I)>0\right\}\right) .
$$

Since we have $g(x)=\inf _{\rho>0} \delta_{\rho}(x)$, it is sufficient to prove that each $\delta_{\rho}$ is a Borel function (see Federer ${ }^{2} 2.2 .15$ ). Let $c \in \mathbb{R}$ and $x \in \operatorname{int} B$; if $\delta_{\rho}(x)>c$, then there exists $I \in \mathcal{C}_{G}$ such that $x \in I$, $\operatorname{diam} I<\rho, \eta(I)>0$ and $\Gamma(I)>c \eta(I)$. This means that $\delta_{\rho}(y)>0$ for every $y \in I$, which is an open set, and so $\delta_{\rho}(] c,+\infty[)$ is an open set; in particular, it is Borel.

For $A \subseteq \mathbb{R}^{n}$ and $\rho>0$, we denote with $\mathcal{N}_{\rho}(A)$ the set

$$
\left\{x \in \mathbb{R}^{n}: d(x, A)<\rho\right\} .
$$

Lemma 6.2. Let $c>0$ and suppose that $K$ is a compact subset of int $B$ such that $K \subseteq\{x: g(x)>c\}$. Then for every $G$-figure $P \in \mathcal{P}_{G}$ such that $K \subseteq P$, we have

$$
c \eta(K) \leq \Gamma(P) .
$$

Proof. Let

$$
\mathcal{C}=\mathcal{C}_{G} \cap\left\{\operatorname{cl} I: I \in \mathcal{I}_{G}, I \cap K \neq \emptyset, I \subseteq P, \eta(I)>0, \frac{\Gamma(I)}{\eta(I)}>c\right\} ;
$$

then $\mathcal{C}$ is fine with respect to $K$. Applying Corollary 6.1, we find a countable disjoint subfamily $\left\{I_{k}: k \in \mathbb{N}\right\} \subseteq \mathcal{I}_{G}$ such that

$$
\left(\bigcup_{k \in \mathbb{N}} I_{k}\right)_{*} \subseteq P, \quad \eta\left(K \backslash\left(\bigcup_{k \in \mathbb{N}} I_{k}\right)_{*}\right)=0 .
$$

For every $l \in \mathbb{N}$ we have

$$
\Gamma(P) \geq \Gamma\left(\left(\bigcup_{k=1}^{l} I_{k}\right)_{*}\right) \geq \sum_{k=1}^{l} \Gamma\left(I_{k}\right) \geq c \sum_{k=1}^{l} \eta\left(I_{k}\right)=c \eta\left(\bigcup_{k=1}^{l} I_{k}\right) .
$$

As $l \rightarrow \infty$, it follows that

$$
\Gamma(P) \geq c \eta\left(\bigcup_{k \in \mathbb{N}} I_{k}\right)=c \eta\left(\left(\bigcup_{k \in \mathbb{N}} I_{k}\right)_{*}\right) \geq c \eta(K),
$$

where we used the fact that $\eta\left(\partial_{*} I_{k}\right)=0$ for every $k \in \mathbb{N}$. 
Now we are ready to prove our main theorem.

Theorem 6.2. Let $\Gamma: \mathcal{A} \rightarrow[0,+\infty)$ be a superadditive function, $G$ a full grid with $\mathcal{P}_{G} \subseteq \mathcal{A}$ and $\eta \in \mathfrak{M}(\operatorname{int} B)$ with $\mathcal{P}_{G} \subseteq \mathcal{M}_{0 \eta}^{\text {loc }}$ and $\Gamma \leq \eta$ on $\mathcal{P}_{G}$. Then there exists a Borel function $g:$ int $B \rightarrow[0,1]$ such that:

$$
\int_{E} g d \eta \leq \Gamma(E)
$$

for every $E \in \mathcal{A}$

(ii) if $f: \operatorname{int} B \rightarrow[0,+\infty)$ is a Borel function satisfying

$$
\forall P \in \mathcal{P}_{G}: \int_{P} f d \eta \leq \Gamma(P),
$$

then $f(x) \leq g(x)$ for $\eta$-almost all $x \in \operatorname{int} B$.

Moreover, such a $g$ is uniquely determined $\eta$-a.e.

Proof. Let $g$ be defined as in (6.1). Let $0<t<1$ and $\varepsilon>0$. For $h \in \mathbb{N}$ consider the sets

$$
E_{h}=\left\{x \in E: t^{h+1}<g(x) \leq t^{h}\right\} ;
$$

since $\eta$ is a regular measure, there exists a sequence $\left(K_{h}\right)$ of compact sets in int $B$ such that $K_{h} \subseteq E_{h}$ and $\eta\left(E_{h} \backslash K_{h}\right) \leq \varepsilon 2^{-h-1}$ for every $h \in \mathbb{N}$; in particular, $\left(K_{h}\right)$ is a disjoint sequence. Then we have

$$
\int_{E} g d \eta=\sum_{h \in \mathbb{N}} \int_{E_{h}} g d \eta \leq \sum_{h \in \mathbb{N}} t^{h} \eta\left(E_{h}\right) \leq \sum_{h=0}^{l} t^{h} \eta\left(E_{h}\right)+\varepsilon \leq \sum_{h=0}^{l} t^{h} \eta\left(K_{h}\right)+2 \varepsilon
$$

for a suitable $l \in \mathbb{N}$.

By Lemma 6.2, for every $h \in \mathbb{N}$ there exists a set $P_{h} \in \mathcal{P}_{G}$ such that $K_{h} \subseteq P_{h}$ and $t^{h+1} \eta\left(K_{h}\right) \leq \Gamma\left(P_{h}\right)$. Setting $d(A, B)=\inf \{|x-y|: x \in A, y \in B\}$, let

$$
\rho=\frac{1}{2} \min \left\{d\left(K_{h}, K_{j}\right): 0 \leq h<j \leq l\right\} ;
$$

since the sets $K_{h}$ are compact and pairwise disjoint, we have $\rho>0$. Without loss of generality, we may assume that $P_{h} \subseteq \mathcal{N}_{\rho}\left(K_{h}\right)$, so that $\left(P_{h}\right)$ is a disjoint sequence. Moreover, by substituting $P_{h}$ with $P_{h} \cap E$, which again belongs to $\mathcal{A}$, we may also assume that $P_{h} \subseteq E$. Hence we can continue the inequalities:

$$
\sum_{h=0}^{l} t^{h} \eta\left(K_{h}\right)+2 \varepsilon \leq t^{-1} \sum_{h=0}^{l} \Gamma\left(P_{h}\right)+2 \varepsilon \leq t^{-1} \Gamma(E)+2 \varepsilon .
$$

Letting $\varepsilon \rightarrow 0^{+}$and $t \rightarrow 1^{-}$, (i) is proved.

Now let $f$ be as in the statement; let $x \in \operatorname{int} B, \rho>0$ and $I \in \mathcal{I}_{G}$ be such that $x \in I, \eta(I)>0$ and $\operatorname{diam} I<\rho$. Since

$$
\frac{1}{\eta(I)} \int_{I} f d \eta \leq \frac{\Gamma(I)}{\eta(I)}
$$

taking the supremum limit as $\rho \rightarrow 0^{+}$the proof is complete. 


\section{Acknowledgment}

The authors wish to thank Marco Degiovanni for very helpful discussions and suggestions, and the referees for useful remarks.

The research of the authors was partially supported by M.U.R.S.T. COFIN2000 project "Modelli matematici per la scienza dei materiali" and Gruppo Nazionale per la Fisica Matematica.

\section{References}

1. M. Degiovanni, A. Marzocchi and A. Musesti, Cauchy fluxes associated with tensor fields having divergence measure, Arch. Rational Mech. Anal. 147 (1999) 197-223.

2. H. Federer, Geometric Measure Theory, Die Grundlehren der Mathematischen Wissenschaften, Vol. 153 (Springer-Verlag, 1969).

3. M. E. Gurtin and W. O. Williams, An axiomatic foundation for continuum thermodynamics, Arch. Rational Mech. Anal. 26 (1967) 83-117.

4. M. E. Gurtin, W. O. Williams and W. P. Ziemer, Geometric measure theory and the axioms of continuum thermodynamics, Arch. Rational Mech. Anal. 92 (1986) 1-22.

5. A. Marzocchi and A. Musesti, Decomposition and integral representation of Cauchy interactions associated with measures, Cont. Mech. Thermodyn. 13 (2001) 149-169.

6. M. Šilhavý, Cauchy's stress theorem and tensor fields with divergences in $L^{p}$, Arch. Rational Mech. Anal. 116 (1991), 223-255.

7. C. A. Truesdell, Rational Thermodynamics (Springer-Verlag, 1984), 2nd edition.

8. W. P. Ziemer, Weakly Differentiable Functions, Graduate Texts in Mathematics, Vol. 120 (Springer-Verlag, 1989). 